\title{
Trabalhonecessário
}

Issn: 1808 - 799X

ano 13, número $22-2015$

\section{LA PEDAGOGÍA DE LA EDUCACIÓN TÉCNICA Y PROFESIONAL EN CUBA: SUS ANTECEDENTES}

Caridad Pérez García ${ }^{1}$

\section{Resumen}

El objetivo es revelar los antecedentes históricos que aportan los fundamentos básicos para el desarrollo de la Pedagogía de la Educación Técnica y Profesional cubana, a partir del análisis, de la sistematización de los conocimientos y valores más representativos planteados por un grupo de virtuosos e ilustres pedagogos cubanos de los siglos pasados. Se analizan las grandes transformaciones acontecidas en la ETP después del triunfo de la Revolución Cubana, basadas en la utilización del método dialéctico materialista y el análisis histórico lógico entre otros, que sirvieron de base a esta rama de la pedagogía.

Palabras claves: Educación Técnica y Profesional; Pedagogía de la Educación Técnica y Profesional; Sistema Educativo Cubano.

\section{Resumo}

O objetivo do artigo é revelar os fundamentos básicos da Pedagogia da Educação Técnica e Profissional cubana, considerando os antecedentes históricos que marcaram seu desenvolvimento. Sistematizamos os principais conhecimentos e valores formulados por um grupo de virtuosos e ilustres pedagogos cubanos dos

\footnotetext{
${ }^{1}$ Profesora Titular de la Universidad de Ciencias Pedagógicas "Enrique José Varona" Dra. en Ciencias Pedagógicas por la Universidad Lenin V. I. Moscú. Rusia. Especialista en Pedagogía-Psicología. Profesora Consultante e investigadora del Centro de Estudios de la Pedagogía Profesional de la Facultad de Ciencias Técnicas de dicha universidad. La Habana, Cuba.karys45@yahoo.es
} 


\section{Trabalhonecessário}

Issn: 1808 - 799X

ano 13, número 22 - 2015

séculos passados e, em seguida, analisamos as grandes transformações ocorridas na ETP depois do triunfo da Revolução Cubana, calcadas no materialismo dialético e na análise histórico-lógico, entre outros, que serviram de base a este ramo da pedagogia.

Palavras-chave: Educação Técnica e Profissional; Pedagogia da Educação Técnica e Profissional; Sistema Educativo Cubano.

A lo largo de los siglos, a la educación se le ha atribuido un papel decisivo en el desarrollo de la sociedad y en particular en la formación del ser humano, pues ella permite la transmisión cultural de generación en generación, así como la formación de valores humanos que garanticen la adecuada incorporación de hombres y mujeres a la vida social.

Cuba posee una rica tradición pedagógica donde las prácticas, las ideas, reflexiones y concepciones sobre la educación de los trabajadores han ocupado, sin dudas, un lugar destacado. Como parte del Sistema Educativo Cubano la Educación Técnica y Profesional está inmersa en este contexto de los procesos formativos de los futuros trabajadores y trabajadoras en correspondencia con las necesidades de la sociedad socialista. La Pedagogía de la ETP constituye hoy una rama de la Pedagogía de gran importancia donde se sustenta el desarrollo de un proceso técnico-profesional esencial para la educación técnicos de la producción y los servicios, de acuerdo con las exigencias contemporáneas de elevar la calidad de la formación de este tipo de profesional.

El análisis de diferentes hechos acontecidos en siglos pasados pauta las influencias reveladoras acerca de los antecedentes más significativos que dieron 


\section{Trabalhonecessário}

Issn: 1808 - 799X

ano 13, número 22 - 2015

lugar, de manera paulatina, a los fundamentos y al sistema categorial que hoy conforman elementos del desarrollo de la Pedagogía de la Educación Técnica y Profesional (ETP) en Cuba. Dichas ideas emergieron de las exigencias del contexto económico, político y social cubano y de las influencias del ámbito mundial en las diferentes épocas; propiciaron las bases teórico-metodológicas sobre las cuales se sustenta el desarrollo actual de esta Pedagogía y de las tendencias esenciales de su movimiento en el futuro.

En este artículo no sería posible abarcar los diferentes proyectos, hechos y figuras que testifican la tradición pedagógica cubana respecto a la educación de trabajadores. Nuestro objetivo fundamental es precisar que el surgimiento y desarrollo de la Pedagogía de la Educación Técnica y Profesional tiene antecedentes teóricos y prácticos que han partido de la necesidad y realidad socioeconómica del país en diferentes etapas y que se refuerzan a partir de 1959 con la Primera Revolución Educacional Cubana (ABREU REGUEIRO, 2004). Consiste en revelar los antecedentes más significativos que han dado lugar al surgimiento de la Pedagogía de la ETP en Cuba, a partir del análisis, de la sistematización de los conocimientos más representativos que resultan de nuestra investigación científica en esta temática.

\section{Trabajo y educación}

Durante los tres primeros siglos de colonización (XV, XVI y XVII), no existían escuelas donde se preparaban trabajadores para diferentes oficios u ocupaciones. Sin embargo, ya en el propio siglo XVI y siglo XVII, el desarrollo de algunos renglones como el tabaco, el azúcar, la producción de alimentos y otros, que eran ocupados por los nativos y los negros esclavos traídos de África, constituyeron 


\section{Trabalhonecessário}

Issn: 1808 - 799X

ano 13 , número $22-2015$

fundamentalmente la principal fuerza productiva. Al crecer la flota y el comercio, surge la manufactura, lo que estimuló el desarrollo de diferentes oficios en áreas como la carpintería, la construcción, la fabricación de tejas y se desarrollan con ello las capas medias de artesanos (ABREU REGUEIRO, 2004, p.27-28). En el caso de la enseñanza / aprendizaje de los oficios "[...] es menester considerar que en los siglos XVI al XVIII la enseñanza de los oficios fue surgiendo a fin de dar respuesta a las necesidades socioeconómicas imperantes en el país" (RIVAS RIVAS; RIVAS RIVAS, 1992, p.8).

El surgimiento de escuelas privadas y escuelas religiosas de diferentes ordenanzas después del descubrimiento y conquista de los españoles, trajo consigo nuevos elementos acerca de la organización de la escuela, independientemente a que esta no respondiera de forma sistematizada a un objetivo determinado. El aprender y el enseñar se caracterizó predominantemente por manifestaciones de distintas formas no escolares de educación. En las escuelas religiosas, en general, se estableció un sistema de trabajo pedagógico dirigido a los jóvenes, fundamentalmente pobres, que incluía no solamente los programas de asignaturas básicas, sino también prácticas y talleres para el ejercício de diversos oficios.

El siglo XVII, en general, se caracterizó por el auge en la actividad artesanal y los oficios, existiendo producciones variadas en una masa de trabajadores urbana calificada, vinculado esto al surgimiento del arte manual, lo que repercutió en desarrollo social y en el establecimiento de determinados oficios que fueron ejercidos por la población más humilde. Ya durante la ilustración reformista cubana en el siglo XVIII, se aportan ideas referidas a la necesidad de la preparación de los trabajadores, de acuerdo con los intereses de la oligarquía criolla, entre ellos Francisco de Arango y Parreño (1765 -1837), el cual se manifiesta a favor de la introducción de la maquinización en Cuba. 


\section{Trabalhonecessário}

Issn: 1808 - 799X

ano 13, número $22-2015$

En 1793 se llevó a cabo la creación de la Sociedad Económica de Amigos del País (SEAP), sociedad que agrupó en su Sección de Artes y Oficios a prestigiosas figuras de la intelectualidad cubana de la época y que brindaron aportes significativos a las ideas y a las prácticas sobre la educación de los trabajadores (ABREU REGUERO, 2004). Miembro de esta Sociedad fue el eminente pedagogo José de la Luz y Caballero (1800 -1862) que dedicó parte de su vida a la educación de la juventud. A él se debe la elaboración de uno de los primeros proyectos sobre cómo debía ser una escuela de nivel medio profesional, el cual se plasma en su Informe sobre la Escuela Náutica o Proyecto sobre el Instituto Cubano, "primer resultado de los esfuerzos de los hacendados e intelectuales cubanos por introducir la Educación Técnica en el país" (CARTALLA COTA, 1989, p.71). En este informe se fundamenta la necesidad de abrir nuevas carreras a la juventud de nuestra patria. Este instituto debía ser una especie de Escuela General de Artes y Oficios, unido a una Escuela Normal para la formación de docentes, por el carácter integral y politécnico del mismo, lo que constituye una de las más avanzadas concepciones que podían plantearse en esa época.

Este proyecto, aunque nunca llegó a materializarse constituyó uno de los primeros aportes científico - teóricos significativos en la historia del surgimiento y desarrollo de la Pedagogía de la ETP en Cuba, ya que en él se evidenciaron antecedentes de concepciones que hoy en día tienen plena vigencia, como por ejemplo:

- La fundamentación del vínculo o integración entre la preparación profesional y la realidad y necesidad del país.

- $\quad$ El carácter nacional de la preparación profesional.

- La relación entre la docencia y la investigación. 


\section{Trabalhonecessário}

Issn: 1808 - 799X

ano 13, número 22 - 2015

- El vínculo o unidad entre teoría y práctica.

- La profesionalización de los contenidos de enseñanza.

- La concepción del profesor integral que imparte diferentes asignaturas afines.

- $\quad$ La actualización científico- técnica de los contenidos.

- La necesidad de una preparación particular, específica para los profesores de este tipo de escuela.

- El carácter continuo de la educación; la necesidad de que el alumno aprenda a estudiar.

- $\quad$ La necesidad de aplicar métodos productivos en la enseñanza.

- $\quad$ La integración de la formación de profesores al instituto, lo que pudiera considerarse un antecedente importante del proceso de Universalización de la Educación Superior Pedagógica. (CARTALA COTA, 1989, p. 71-72).

A mediados del siglo XIX, la destacada figura de José Martí (1853 - 1895) constituye uno de los exponentes más significativos del pensamiento político-social de la época. Son muy amplios y multifacéticos los análisis del Héroe Nacional sobre diferentes ámbitos de la vida social y económica, a lo cual no escapa su visión respecto a la ciencia y la técnica y el lugar de la educación de los seres humanos en la misma. Baste recordar, entre otros, sus posiciones sobre la enseñanza científica, el lugar de los conocimientos científicos y técnicos en la educación del hombre integral, su cultura general; su concepción dialéctica respecto a la unidad teoríapráctica, hombre-actividad económica, hombre-máquina, hombre-naturaleza, conocimientos técnicos-instrucción general (ABREU REGUERO, 2004, p.30-31) las cuales mantienen una vigencia total en las concepciones pedagógicas del sistema educativo cubano. 


\section{Trabalhonecessário}

Issn: 1808 - 799X

ano 13, número 22 - 2015

Muy importantes son también sus análisis sobre la necesidad de instruir al trabajador del campo, tarea encomendada a 'Maestros Ambulantes', los que personalmente atenderían a estos campesinos, directamente en su puesto de trabajo, el campo, instruyéndolos en los adelantos de la ciencia y la técnica, lo que hoy se denominaría educación continua del trabajador, en su puesto mismo de trabajo. (ABREU REGUERO, 2004, p.12).

Estos, entre otros aspectos, abordados por el Apóstol José Martí, en su vasta obra, demuestran la presencia en la misma de muchos de los presupuestos que sustentan hoy el desarrollo de una Pedagogía de la ETP en Cuba. En especial, se destaca su visión sobre la Educación General y Técnico-Profesional del trabajador, su cultura general mediante la unidad del estudio con el trabajo, de la educación con la actividad económica; concepción de profundas raíces humanistas y revolucionarias, que está presente en la materialización de la ETP cubana actual y en los postulados de su Pedagogía.

Significativos en esta etapa fueron también los aportes del ilustre pedagogo cubano Enrique José Varona (1849-1933), el que defendió, entre otros muchos aspectos, la necesidad del vínculo entre instrucción general y técnico - profesional, la importancia de la primera como base de la segunda, el valor de la aplicación de la ciencia y la investigación a la enseñanza técnica. Al respecto enfatizaba:

"[...] la instrucción general, aunque sirve por sí misma para el desarrollo y la cultura del espíritu, es una preparación para la enseñanza profesional... el complemento necesario de una buena instrucción general es la difusión de la enseñanza técnica en todos sus grados, desde los oficios manuales hasta las profesiones llamadas liberales" (VARONA, 1961, p. 17).

No pueden estos apuntes olvidar el papel de Fernando Aguado y Rico (1859 1941) en el desarrollo de la Educación Técnica y Profesional y su Pedagogía, el que 


\section{Trabalhonecessário}

Issn: 1808 - 799X

ano 13 , número $22-2015$

fundara en 1882 la Escuela Preparatoria de Artes y Oficios, para la educación técnica industrial, escuela que contribuyó notablemente a la formación de trabajadores en el siglo XX.

Aguado y Rico no escribió su obra, pero sus ideas las puso, en su gran mayoría, en práctica durante los 48 años de vida que dedicó a la Educación Técnica y Profesional. Se destacó en el perfeccionamiento de este tipo de Educación, en la organización de cursos para trabajadores y en sus aspiraciones de preparar profesores para estas escuelas. La Escuela de Artes y Oficios se considera que marcó el inicio del desarrollo de la ETP en Cuba, pero existen otros antecedentes de gran valor para esta Pedagogía.

Fernando Aguado y Rico proyectó, unido a la escuela, un "Conservatorio Nacional de Artes y Oficios" destinado a la formación del profesorado de la Escuela Técnica Industrial, como coronamiento de la cultura industrial.

"Este conservatorio era una cátedra en la que sus profesores,
escogidos entre los más notables especialistas, prepararán para el
profesorado de las Escuelas Técnicas Industriales de todos los
grados. Sus lecciones serán explicadas en lenguaje sencillo y
fácilmente asimilable para todos los oyentes. A ellas deberán tener
acceso también los jefes de taller e industrias, empleados técnicos,
obreros y cuantos se interesen por la industria y el engrandecimiento
de la vida económica del país" (ABREU REGUEIRO, 2004, p.32).

Los Reglamentos y otros documentos de estas instituciones, muchos de los cuales aún se conservan, constituyen un antecedente teórico de extraordinario valor de los fundamentos de una Pedagogía de la ETP, documentos poco estudiados y valorados en su vigencia. Por ejemplo: la necesidad de que el profesor de este tipo de educación sea ante todo un buen técnico, un técnico pedagogo, la necesidad de preparar a los directivos y técnicos de la producción en elementos pedagógicos, pues 


\section{Trabalhonecessário}

Issn: 1808 - 799X

ano 13 , número $22-2015$

"nosotros creemos que para hacer un aprendizaje en un taller industrial cualquiera, no basta solamente con saber hacer, sino también saber tratar, saber guiar, saber mejorar al futuro aprendiz" (ABREU REGUEIRO, 2004, p.32).

En resumen, durante los siglos de colonización se aprecian diferentes concepciones pedagógicas, fundamentalmente en forma de reglas para el trabajo en la práctica de los oficios relacionadas con los perfiles técnicos, en estrecha interrelación con el surgimiento y desarrollo de este tipo de Educación en Cuba, que constituyen rasgos de una incipiente Pedagogía de la ETP, sustentada en la necesidad de la preparación del trabajador que se exigía, en la necesidad histórica de cambiar la realidad social y al hombre.

En la investigación que realizamos se han puesto de manifiesto conceptos y postulados que han identificado a la ETP y en especial a sus fundamentos teóricopedagógicos desde sus inicios en el país, los que se relacionan directamente con:

- $\quad$ La fundamentación del profesor de la ETP como un buen técnico.

- Las particularidades del alumno de la ETP, su posición social y particularidades psicológicas.

- $\quad$ La necesidad de la formación integral del trabajador.

- La unidad entre formación general y formación técnico- profesional.

- La unidad de la ETP con la producción, con la economía.

- La introducción de la nueva técnica y la preparación del trabajador para dominarla.

- La profesionalización de los contenidos de la ETP, entre otros.

Dentro de estos aspectos se observa la utilización de conceptos como Enseñanza de Oficios, Aprendiz, Taller, Escuela de Artes y Oficios, Escuela Profesional, Maestro Agrícola, Conservatorio Nacional de Artes y Oficios, entre 


\section{Trabalhonecessário}

Issn: $1808-799 X$

ano 13, número $22-2015$

muchos otros. Son conceptos que en su esencia están directa o indirectamente contenidos en los componentes de los modelos que se proponen en algunas de las investigaciones realizadas en la Universidad de Ciencias Pedagógicas Héctor Alfredo Pineda Zaldívar (ABREU REGUEIRO, 2004, p.15), que aportan fundamentos pedagógicos actuales que sustentan y amplían los procesos de educación técnica y profesional.

\section{De la República a la Revolución}

Durante la República, en sus 56 años de existencia, la Educación Técnica y Profesional constituyó uno de los sectores de la educación más atrasados y apartado de las necesidades reales del país. Ello ocasionó contradicciones, dadas entre las necesidades reales de desarrollo de la ETP y las limitaciones, no solo con un número limitado de centros y de contenidos de baja calidad, sino también por no contar con una preparación mínima de sus profesores, de acuerdo a las exigencias de las empresas cubanas y extranjeras respecto la base de la explotación de los trabajadores.

A pesar de esto fueron naciendo diferentes leyes y documentos sobre la Educación Técnica y Profesional, se crearon algunas Escuelas Técnicas Industriales y otros tipos de centros educacionales para la preparación de los trabajadores, lo que respondía en la mayoría de los casos a fines políticos nacionales y locales y no a una estrategia de educación en función de las reales necesidades socio-económicas del pueblo cubano.

Una obra de indiscutible valor en esta etapa lo constituye el libro "Un siglo de enseñanza industrial en Cuba", del profesor de la Escuela Superior de Artes y 


\section{Trabalhonecessário}

Issn: 1808 - 799X

ano 13, número 22 - 2015

Oficios, José Montó Sotolongo, publicado en 1951 en el que se compilan y fundamentan aspectos que, sin dudas reflejan particularidades de una Pedagogía propia de la ETP. Este libro recoge definiciones de conceptos propios de esta rama de la Pedagogía, referentes sobre la historia de este tipo de Educación, sobre la enseñanza práctica y su metodología, la enseñanza industrial, la formación de profesores para este tipo de escuelas, sobre experiencias en otros países, entre otros aspectos.

Se debe señalar el papel jugado por los Estados Unidos en la educación cubana durante la etapa prerrevolucionaria, cuestión que se aprecia también en el campo de la ETP, en especial en sus intereses por dirigir la preparación de los profesores, aspecto que se corrobora en la publicación por la Organización de Estados Americanos (OEA), en 1958, de los tres primeros folletos de una serie destinada a ello y que responden a los títulos de:

- Metodología de la Enseñanza Práctica.

- Psicología aplicada a la Educación Técnica.

- $\quad$ Organización y Administración de talleres escolares.

Estos folletos hacen referencia a la necesidad de la preparación pedagógica de estos profesores, a principios y prácticas útiles que han de guiar a la educación técnica moderna en los Estados Unidos de América, obra original en inglés con el título: "The instructor and his job" y fue editada por la Universidad del Estado de Nueva York, constituyendo un resultado de las experiencias en ese país y como etiqueta se recomienda atender a las condiciones específicas de cada lugar a la hora de aplicarlos. Aunque de forma muy general, brindan elementos importantes sobre el papel central del profesor de la educación técnica y sus relaciones respecto al 


\section{Trabalhonecessário}

Issn: 1808 - 799X

ano 13 , número $22-2015$

ingeniero y el artesano, así como fundamentos generales para su trabajo, constituyen sin dudas antecedentes de la Pedagogía de la ETP.

Durante la República Mediatizada, pese a la imposición de programas educacionales en función de la expansión ideológica y económica de Estados Unidos y los ambiciosos y no materializados objetivos declarados de la ETP, se ponen de manifiesto también diferentes ideas, que dando continuidad, en muchos casos a criterios anteriores y centrados en los esfuerzos por buscar soluciones a la práctica, demuestran rasgos distintivos, en función de una Pedagogía propia de este tipo de educación. Los criterios están relacionados con:

- $\quad$ El desarrollo de una Didáctica de la ETP, como fundamento para la dirección de la enseñanza y la formación de maestros para este tipo de escuelas.

- $\quad$ El desarrollo de Metodologías de la Enseñanza de las asignaturas técnico - profesionales.

- $\quad$ Se reafirma la necesidad de formar a un profesor de la ETP de manera específica, considerándose para ello una enseñanza de cuarto grado, lo cual nunca se puso en vigor.

- $\quad$ Se fundamentan pedagógicamente los nuevos planes y programas de estudio, lo que no llega a consumarse en su plenitud.

- Se elaboran reglamentos y otros documentos de extraordinario valor teórico para la dirección de este tipo de Educación en las diferentes instituciones.

- $\quad$ Se introduce la ETP programada por radio, para formar técnicos en diferentes especialidades, actividades poco estudiadas y que por su valor teóricometodológico constituyen un fundamento importante para la Pedagogía de la ETP, por su impacto y su vigencia en los nuevos programas educacionales que se acometen en la actualidad. 


\section{Trabalhonecessário}

Issn: 1808 - 799X

ano 13 , número $22-2015$

- $\quad$ Surgen concepciones en cuya base se encuentran un gran número de conceptos, por ejemplo: Escuela de Artes y Oficios, Arte Manual, Artes Industriales, Estudios Domésticos, Escuela Técnica Industrial, Enseñanza Politécnica, Escuela Politécnica, Escuela Técnica Industrial, Profesor de enseñanza teórica y práctica, Taller, Fábrica, Campo de Aplicación, Obrero Calificado, Maestro de Obra, Instructor de Oficio, Centro Tecnológico.

Estos conceptos e ideas, que dan continuidad a muchos de los abordados en la etapa colonial constituyen soporte teórico y antecedente esencial del modelo actual de la Pedagogía de la ETP.

Durante esta etapa el magisterio perteneciente a este tipo de educación, digno continuador de las mejores tradiciones pedagógicas cubanas, comprendió la significación de su organización para la lucha revolucionaria. Existieron muchos ejemplos dignos, valientes y abnegados de vinculación y unidad con el movimiento obrero cubano, de oposición a la politiquería de turno, de defensa de los derechos del magisterio, que con su obra lograron dentro del régimen existente el surgimiento y desarrollo del germen de una Educación Técnica y Profesional nueva. Se trataba de una Pedagogía Proletaria que triunfó el primero de enero de 1959, momento en que el Gobierno Revolucionario hizo realidad el Programa del Moncada: desarrollar una reforma integral en el Sistema Nacional de Educación que permitiera sentar las bases para producir un salto cualitativo y cuantitativo en la preparación de trabajadores y trabajadoras capaces de llevar adelante la economía del país.

En correspondencia con los nuevos planes de industrialización y desarrollo que generaba la Revolución, la ETP recibió un extraordinario impulso:

"Esto tiene mayor importancia en un país como Cuba que está esforzándose en dejar atrás las formas atrasadas de su economía. Si precisamente una de las mayores preocupaciones 


\section{Trabalhonecessário}

Issn: 1808 - 799X

ano 13 , número $22-2015$

del gobernante de hoy es acelerar el proceso de preparación de técnicos, pues la Reforma Agraria ya en marcha, traerá inexorablemente el desarrollo industrial y acrecentará el potencial económico de nuestra patria en un término más corto de lo que muchos piensan" (RAMíREZ DE LA CRUZ, 1959, p. $5)$.

Las palabras anteriormente citadas del Dr. Orlando de la Cruz Ramírez, director del Centro Tecnológico Frank País, de Ceiba del Agua, en la temprana fecha del 30 de Julio de 1959, sobre la reestructuración de la Escuela Técnica, constituye una muestra fehaciente de la nueva concepción de la ETP y de su Pedagogía, cuestiones que hoy están presentes en el proceso de perfeccionamiento de este tipo de Educación.

De especial importancia y visión constituyen en este discurso las bases que se plantean para realizar una adecuada estructuración de la Educación Técnica donde se destacan, entre otras: a) la ubicación de las escuelas en zonas productivas que lo requieran y que respondan al carácter profesional que exija esta zona; b) la coordinación con los centros de producción en función de la docencia; c) que los planes de estudio y los programas se adapten a los principios didácticos básicos de este tipo de educación, orientándolos de acuerdo a los requisitos del Proceso Revolucionario Cubano y d) establecimiento de la investigación profesional.

Se crearon las cooperativas de producción en cada centro. Sin necesidad de profundizar, en estas bases están contenidas las líneas directrices que han guiado el desarrollo de la ETP desde el inicio del proceso revolucionario y los fundamentos en los cuales se debía apoyar su pedagogía.

La creación de una ETP nueva y en correspondencia con las exigencias y necesidades del socialismo no fue tarea fácil. Faltaban experiencias, escuelas, 


\section{Trabalhonecessário}

Issn: 1808 - 799X

ano 13, número 22 - 2015

talleres, laboratorios, maestros, materiales didácticos, literatura y sobre todo planes de estudio y programas que se ajustaran a la nueva realidad.

Luego de los grandes y profundos cambios producidos en 1959 en el Sistema Nacional de Educación y en especial en la ETP, se pudieron analizar, de forma general, cuatro grandes etapas de transformaciones y desarrollo en este tipo de Educación y su Pedagogía, las cuales coinciden con las décadas de los años 1960 hasta 1990, esta última llegando hasta el año 2004.

Según el Censo de Población efectuado en 1953, en el país existían cerca de dos millones de analfabetos, lo que representaba aproximadamente el $23 \%$ de la población, destacándose como sectores con mayor analfabetismo el agrario, el de los desempleados y los obreros pobres. El inicio de la obra transformadora de la Revolución Cubana exigía una campaña que erradicara este problema, como vía que proporcionara al pueblo y en especial a las capas más humildes del mismo, el dominio de la ciencia, la técnica y la cultura. Fue una campaña cuyos efectos y consecuencias se encuentran en la base del desarrollo actual alcanzado por de la educación cubana.

La década de los años 1960 constituyó, además, una etapa decisiva en el desarrollo de la ETP, aportando singulares experiencias que marcaron para siempre a este tipo de Educación. Los profesores revolucionarios, procedentes en gran parte de fábricas y centros de trabajo, jugaron un papel destacado en la formación práctica de sus alumnos en talleres y áreas de producción, al transmitir sus hábitos y tradiciones obreras. Fue una década de creación de nuevas Escuelas Técnicas Industriales en diferentes provincias, así como en fábricas; de reconstrucción de otras, de readaptación de locales, de nacionalización, de preparación masiva de profesores a partir de obreros; de desarrollo de cursos de mínimo técnico para elevar el nivel del trabajador en su puesto de trabajo. 


\section{Trabalhonecessário}

Issn: 1808 - 799X

ano 13, número 22 - 2015

En esos años, por iniciativa de Fidel Castro, se crearon varias Ciudades Agrarias para la preparación de cuadros agrícolas. Se creó el Instituto Pedagógico Industrial (IPI), el cual constituyó un primer intento materializado de institución para la preparación de profesores de la ETP. Se produjeron grandes modificaciones en los planes y programas de estudios, se inició el asesoramiento por parte del campo socialista europeo a la ETP, se creó, por iniciativa de Fidel, el Consejo del Plan de la Enseñanza Tecnológica, la formación masiva de técnicos para la agricultura, la combinación de la formación técnica con la preparación militar en el Viceministerio de Enseñanza Tecnológica Militar (VETM).

Se logró la participación determinante de la ETP en tareas productivas, se introdujo la Pasantía o Práctica Pre-profesional, con una determinante función educativa y de reafirmación profesional, aleccionadora experiencia formativa, que además de constituir un valioso aporte al sistema educativo moderno y revolucionario, posibilitó que los alumnos tecnológicos ayudaran a la producción, desde un año antes de su graduación. Asimismo, el método adoptado para planear la Pasantía y su organización constituyen un paso pedagógico de incalculable estima en la unidad de la teoría y la práctica.

Significativas fueron también las diferentes tareas acometidas por el Consejo del Plan de la Enseñanza Tecnológica en relación con la divulgación de los conocimientos técnicos. Muestra de ello fue la creación del Instituto Tecnológico Popular (ITP), a través de la Televisión y de ediciones de folletos de apoyo, así como lecciones en los periódicos Juventud Rebelde y El Mundo. No menos importantes fue también la participación en largas zafras del pueblo y maniobras militares, bajo condiciones severas de trabajo y disciplina militar.

Esta etapa de intenso "quehacer educativo", no exento de deficiencias e insuficiencias debido a las condiciones políticas, económicas y sociales que se dan 


\section{Trabalhonecessário}

Issn: 1808 - 799X

ano 13 , número $22-2015$

en ella, dejaron una huella imborrable en la fortaleza político-ideológica, de moral revolucionaria, de patriotismo y de sacrificio por la Revolución en sus participantes, que los distinguen y enorgullecen hoy donde quiera que se encuentren, punto de referencia de las virtudes que deben inculcarse a los jóvenes de hoy (BERNAL, R. 1987).

Al respecto, en diciembre de 1966, el Comandante Fidel Castro señaló: "Lo que nos interesa no es solo formar técnicos, sino técnicos integrales, ciudadanos mejores. Y que si tenemos urgencia de técnicos, ¡siempre será más urgente formar hombres verdaderos, formar patriotas, formar revolucionarios!".

Este período de surgimiento y desarrollo de una ETP revolucionaria llevó implícito el cambio y la profundización en sus fundamentos pedagógicos, en estos primeros años algo empíricos, pero sin dudas efectivos en muchos sentidos y de obligatorio análisis para derivar de ellos las mejores concepciones y prácticas para la educación del trabajador de hoy. Esta etapa, aunque poco estudiada desde el punto de vista teórico, permite ya la delimitación inicial de un cuerpo de conceptos, regularidades y principios que acreditan a la Pedagogía de la ETP como un campo especial dentro de la Pedagogía Revolucionaria en Cuba y que han permitido su consolidación en las décadas posteriores hasta la actualidad.

En la década de 1960 se introducen y desarrollan un sin número de nuevos conceptos que revolucionan a la ETP, dentro de los cuales se distinguen: la competencia laboral, integración Industria-áreas docentes de producción, mínimo técnico, formación práctica, ciudades agrarias, orientación profesional, operarios especializados, graduado competente, superación profesional de profesores, prácticas en industrias y superación de graduados.

Por su parte, la década de los años 1970 constituyó una etapa de florecimiento de la ETP con grandes y profundos cambios dirigidos a su 


\section{Trabalhonecessário}

Issn: 1808 - 799X

ano 13 , número $22-2015$

consolidación. Se suscitó una extraordinaria expansión que provocó en cierta medida un descenso en la eficiencia del proceso educativo. A la vez estimuló la estabilización de este tipo de educación, la cual a principios de esta década se oficializa e institucionaliza en el marco del proceso de perfeccionamiento del Sistema Nacional de Educación como Subsistema de Educación Técnica y Profesional, con un carácter integrador, lo que la sitúa en su justo lugar de acuerdo a las funciones a desempeñar en la sociedad cubana.

En esta etapa se acentúa la colaboración con el campo socialista, en especial con la URSS en la habilitación de talleres y laboratorios; se crean numerosos Centros de Formación Profesional en diferentes Ministerios; se introduce una nueva estructura de especialidades, así como nuevos planes y programas de estudio, todo ello ajustado a las nuevas necesidades socio-económicas del país. Se realiza el Primer Congreso del Partido Comunista de Cuba, en 1975, que traza la ruta a seguir en el desarrollo de la educación en el país y en especial de la ETP:

"Proporcionar a las nuevas generaciones la adecuada formación en el aspecto político, intelectual, científico, técnico, físico, estético, politécnico, laboral y patriótico-militar, a la par de una preparación profesional en la especialidad correspondiente, de modo que la sociedad pueda contar, en las cantidades necesarias y con la calidad requerida, con los obreros, técnicos de nivel medio... aptos para el disfrute de su pleno bienestar humano, como para responder a las demandas del desarrollo económico y social del país, en los futuros años del presente y próximo siglo." (PRIMER CONGRESO, 1975, P.368).

Momento determinante en esta etapa lo constituyó la promulgación de la ley 1272 de 1974 sobre la Capacitación Técnica y Profesional de los trabajadores, la que trazó una política única en la preparación de los trabajadores tanto en centros de la ETP como en las entidades productivas. Esta ley subrayó también la necesidad de 


\section{Trabalhonecessário}

Issn: 1808 - 799X

ano 13 , número $22-2015$

organizar la investigación científica en este tipo de educación y la introducción de los logros de la ciencia y la técnica, así como las experiencias pedagógicas avanzadas, tanto en la formación inicial como en la superación de los trabajadores. Esta fue una vía fundamental para elevar la calidad de esta preparación de acuerdo a las exigencias de la economía y la Revolución Científico Técnica.

La década de los años 1970 se destacó, además, por la creación del Instituto Pedagógico para la Educación Técnica y Profesional (IPETP) por Resolución Ministerial No. 210 de 1973, el cual asume la tarea de la formación y superación profesional pedagógica de los profesores y directivos de este tipo de educación. A partir de este momento inicia un fuerte trabajo científico-investigativo en busca de las mejores concepciones y prácticas para impulsar la ETP en el país.

Es importante subrayar que el IPETP, denominado posteriormente Instituto Superior Pedagógico para la Educación Técnica y Profesional, hoy Universidad de Ciencias pedagógicas Héctor Alfredo Pineda Zaldívar, ha constituido el principal promotor del desarrollo de la Pedagogía de la ETP, materializado en una amplia labor científico-investigativa, cuyos resultados se introducen sistemáticamente en la práctica. Es una institución que lidera las investigaciones fundamentales con relación a la consolidación de una Pedagogía de este tipo de educación y que forma y supera a los profesores y directivos en estos contenidos. En estos años se creó también el Destacamento Pedagógico de la ETP con estudiantes seleccionados.

En resumen, esta etapa de florecimiento de la ETP se caracterizó ,desde el punto de vista pedagógico, por la ampliación y profundización en el estudio de los fundamentos que deben sustentar su perfeccionamiento, aspectos contenidos en los planes y programas de estudio y en los documentos normativos elaborados, así como en una amplia gama de publicaciones, que aunque se apoyan en experiencias y estudios realizados en países socialistas europeos, tienen el valor de ser vistos y 


\section{Trabalhonecessário}

Issn: 1808 - 799X

ano 13, número $22-2015$

ajustados a la realidad cubana, por lo que es necesario reconocer los aportes que brindan a la Pedagogía en Cuba y su plena vigencia. Entre estos se pueden mencionar los materiales de carácter normativo - metodológicos editados para los Seminarios Nacionales a Dirigentes, Metodólogos e Inspectores de las Direcciones Provinciales y Municipales de Educación, los que poseen un extraordinario valor teórico-pedagógico en tanto aportan conceptos, regularidades y principios esenciales a la Pedagogía de la ETP que continúan enriqueciendo los fundamentos teóricos de esta rama de la Pedagogía.

Por su parte, los años 1980 marcaron un periodo de consolidación de la ETP y de introducción de cambios sustanciales y nuevas concepciones pedagógicas, acorde a la nueva realidad socioeconómica del país y a los retos internacionales al respecto. La red de Centros de la ETP se eleva a 463 Escuelas Politécnicas y 150 Escuelas de Oficio.

Se acometió el perfeccionamiento de la estructura de especialidades, la cual se distinguió por una reducción e integración de las mismas, ajustándose a las necesidades de la producción, paso fundamental para el ulterior desarrollo del Subsistema. Se aplica la concepción de preparar especialistas de 'perfil amplio' y 'doble perfil', con el objetivo de lograr una educación más integral y garantizar el desempeño de un mayor número de ocupaciones por el egresado y por tanto una flexibilización en la ubicación laboral para dar respuesta a la demanda de trabajadores calificados para la producción.

Es un periodo de intensificación en la preparación de directivos a través de Seminarios Nacionales, cursos y otras modalidades de superación. Son años donde se establecen acuerdos importantes como el 1941 del Comité Ejecutivo del Consejo de Ministros, que oficializa la colaboración Escuela politécnica - Entidad Productiva en relación con la educación técnica y profesional del trabajador, y que amplía, 


\section{Trabalhonecessário}

Issn: 1808 - 799X

ano 13 , número $22-2015$

precisamente, lo planteado por el Acuerdo 356 de 1976 sobre el apoyo de los organismos a la ETP.

La década de los años 1980 se destacó también en el trabajo científicoinvestigativo, desarrollándose una amplia gama de investigaciones en el ámbito de la Pedagogía de la ETP. aunque desde el punto de vista teórico tenían como mérito fundamental la sistematización y la orientación para la práctica, brindaron aportes importantes que estimularon las transformaciones en este tipo de educación; investigaciones relacionadas con las particularidades del proceso educativo en los Centros de la ETP y el perfeccionamiento de este tipo de educación en el país.

Significativo también en esta década fue el que se comenzara a hablar y trabajar en función de la consolidación de una Pedagogía de la ETP, en términos de una Pedagogía Profesional. Se inician investigaciones teóricas sobre este campo en el ISPETP, así como el debate científico al respecto, se desarrollan cursos cortos sobre los resultados que se van obteniendo, así como otros impartidos por especialistas de la República Democrática Alemana y la URSS, se comienzan a presentar trabajos sobre este tema en diferentes eventos científicos nacionales e internacionales.

Fueron años de enriquecimiento conceptual en relación con este tipo de educación y con su pedagogía; enriquecimiento no exento de traspolaciones indiscriminadas de otros contextos y uso indistinto de conceptos, cuestión que, aunque afectó el entendimiento y la tradición teórico-conceptual nacional, permitió ampliar la visión sobre este campo teórico y comprender un poco más la necesidad de la inserción de la Educación Técnica y Profesional en el contexto internacional. 


\section{Trabalhonecessário}

Issn: 1808 - 799X

ano 13, número $22-2015$

\section{Avances en el "período especial en tiempos de paz"}

Este periodo de consolidación de la ETP concluye con un hecho que ejerció un impacto negativo: la caída del campo socialista europeo, lo cual afectó en mayor o menor medida a todos los sectores socio-económicos del país, no estando exento de ello el Sistema Nacional de Educación y en especial la ETP, dadas las particularidades del proceso pedagógico que desarrolla y los recursos que necesita para su ejecución.

El recrudecimiento del bloqueo norteamericano hacia Cuba trajo como consecuencia el establecimiento de un "período especial en tiempo de paz" que, independientemente de las afectaciones que provocó en el Subsistema de la ETP permitió, sobre la base de la labor digna y abnegada de sus pedagogos, alcanzar los objetivos propuestos con un máximo de calidad. No se cerró ninguna Escuela Politécnica, se estableció una nueva estructura de especialidades, se reajustaron los planes de estudio y programas buscando la integración y flexibilidad en los mismos, se volcó la actividad práctica hacia las Entidades Productivas, lográndose una más eficiente integración entre Escuela Politécnica / Entidad Productiva. Se ha descentralizado la matrícula, profesionalizado los contenidos y el proceso en general. También se introduce la Metodología de la Investigación Científica en el proceso de educación, integrándose a la docencia y la producción y se elevan, además, las exigencias hacia la superación continua tanto de profesores como directivos.

Esta etapa, pese a las limitaciones provocadas a este tipo de Educación, estimuló la búsqueda de nuevas alternativas, se diversificaron las modalidades de superación académica, se amplió la producción científica en cuanto a literatura pedagógica, desarrollo de eventos científicos y publicaciones en las diferentes 


\section{Trabalhonecessário}

Issn: 1808 - 799X

ano 13 , número $22-2015$

vertientes de la Pedagogía de la ETP, en especial en las Metodologías de la Enseñanza de las ramas técnicas.

Por otra parte, este periodo permitió la toma de decisiones y con ello el inicio del reordenamiento económico, la recuperación y reanimación de la producción, el redimensionamiento y perfeccionamiento empresarial socialista. Estos cambios organizacionales y la renovación técnica situaron al hombre, al trabajador, como centro de los procesos antes mencionados, exigiendo de ellos una adecuada preparación. Para ello, las empresas socialistas tuvieron que asumir una alta responsabilidad en la superación de los recursos humanos. La ETP y su Pedagogía pasan a jugar un papel determinante en la producción.

Todos los cambios producidos tienden, cada vez más, a la mayor unidad de pensamiento y acción pedagógica entre la Escuela Politécnica y la Entidad Productiva, área donde la Pedagogía de la ETP posee un amplio campo de investigación.

Pese a los problemas ya enumerados, la década de los años 1990, del Período Especial y de la Batalla de Ideas, constituyó una década determinante en el proceso de consolidación de una Pedagogía de la ETP en Cuba. Etapa de fundación del Centro de Estudios de la Pedagogía Profesional (CEPROF), el cual asume como tareas fundamentales: el desarrollo de un modelo teórico de la Pedagogía de la ETP, donde se expresaron los principales conceptos, categorías, leyes, regularidades y principios con enfoques actualizados, cuyos aportes facilitaran la realización de otras investigaciones; la elaboración de un modelo de Escuela Politécnica Cubana que fuese introducido en todo el país, así como el desarrollo de investigaciones dirigidas a la preparación de los profesores para este Subsistema de Educación. Este momento puede considerarse el inicio de la sistematización teórica y del desarrollo de la Pedagogía de la ETP como rama de la Pedagogía en las condiciones cubanas. 


\section{Trabalhonecessário}

Issn: 1808 - 799X

ano 13 , número $22-2015$

Los resultados obtenidos en las investigaciones desarrolladas por el CEPROF y por otros departamentos de la Universidad de Ciencias Pedagógicas Héctor Alfredo Pineda Zaldívar se introducen también en la Educación Técnica y Profesional en productos tales como: Cursos, Asesorías, Diplomados, Maestría en Pedagogía Profesional; esta última con un número significativo de egresados, cuyos trabajos de tesis constituyeron aportes valiosos a los fundamentos de la Pedagogía de la ETP, por sus aplicaciones en las condiciones actuales de desarrollo de esta enseñanza.

También se da inicio a la realización de Talleres Nacionales y Simposios Iberoamericanos auspiciados por este Centro de Estudios sobre Pedagogía Profesional y en especial sobre la Pedagogía de la ETP. Esta fue una etapa de amplias reflexiones y discusiones científicas sobre la temática, sus problemas actuales y perspectivos, las nuevas concepciones teórico-metodológicas y la diversificación de la Educación Técnica y Profesional.

El trabajo de introducción de 'perfiles amplios' en los planes de estudio de la Educación Técnica y Profesional y las nuevas concepciones para el mejoramiento en la calidad en la formación de los obreros que se preparan en las escuelas politécnicas, han sido temas de mucha relevancia después del triunfo de la Revolución Cubana, lo que ha originado diferentes momentos de análisis y cambios necesarios que se han proyectado hacia el mejoramiento de una cultura general integral en la formación del obrero.

Los años de la década del 2000 y hasta la fecha, amplían los fundamentos teóricos de la Pedagogía de la ETP, ocurriendo un cambio de concepción muy profundo, tanto en lo curricular, como en lo mental. Así que las Escuelas Politécnicas se integran plenamente a la Universidad Pedagógica en una misma sede; se materializa en gran medida la concepción que fundamentó Fernando Aguado y Rico de llevar la preparación de los profesores a la Escuela (Unidad de la Escuela de 


\section{Trabalhonecessário}

Issn: 1808 - 799X

ano 13 , número $22-2015$

Artes y Oficios con un Conservatorio para la preparación de los profesores de estas escuelas). Es decir, la preparación directa del profesor de la ETP se trabaja desde su propio rol profesional, vinculando la teoría y la práctica continua y de esta manera se logra la consolidación en el área de la Educación Técnica y Profesional.

Se realiza un importante grupo de investigaciones en la Universidad de Ciencias Pedagógicas Héctor Alfredo Pineda Zaldívar, cuyos resultados enriquecen los sustentos teóricos de la Pedagogía de la ETP. Entre estos se puede mencionar:

$>\quad$ El modelo de Escuela Politécnica Cubana que da continuidad al Modelo de Instituto Politécnico Agropecuario propuesto por la Dra. M. del R. Patiño y otros en el año 1995.

$>$ El modelo de Instituto Politécnico de Informática (MIPI) y sus fundamentos teóricos.

$>\quad$ El modelo educativo integral para el crecimiento personal (MEICREP) y la estrategia metodológica para su aplicación, dirigido al estudio del crecimiento personal del estudiante en situaciones diseñadas del proceso de enseñanzaaprendizaje, a la caracterización del aprendizaje formativo como proceso propiciador del desarrollo integral de la personalidad.

$>\quad$ El modelo de la Pedagogía para la Educación Técnica y Profesional (ABREU REGUEIRO, 2004).

$>\quad$ El modelo de integración de Escuela Politécnica-mundo laboral (LEÓN GARCÍA, 2003).

$>\quad$ El modelo para la práctica laboral por proyectos informáticos.

$>$ El modelo pedagógico para el mejoramiento del desempeño pedagógico profesional de los profesores de Agronomía de los Institutos Politécnicos Agropecuarios. 


\section{Trabalhonecessário}

Issn: 1808 - 799X

ano 13, número $22-2015$

> El modelo para el proceso de diagnóstico integral del estudiante de la Educación Técnica y Profesional.

> La concepción del Profesor General Integral de la Educación Técnica y Profesional; la estrategia para el perfeccionamiento de su desempeño profesional.

$>$ El modelo de los procesos formativos y la estrategia para su implementación en la institución escolar.

$>$ El modelo del proceso de enseñanza-aprendizaje formativo (BERMUDEZ, PÉREZ, 2010) y para la dirección del proceso de enseñanzaaprendizaje en la Educación Técnica y Profesional.

$>\quad$ El modelo para la formación de una estrategia general de aprendizaje y para la formación de proyectos de vida profesionales en las especialidades técnicas.

\section{Conclusión}

En este artículo se analizaron los principales presupuestos que constituyen la base del surgimiento de las ideas pedagógicas que, como antecedentes dieron lugar a una Pedagogía de la Educación Técnica y Profesional (ETP) en Cuba, vinculada siempre a las necesidades del desarrollo socioeconómico del país. Se expuso un análisis de las manifestaciones principales de la educación durante los tres siglos de la colonización, la república y luego de los grandes y profundos cambios producidos desde 1959 en el Sistema Nacional de Educación y en especial en la ETP, de forma general en cuatro grandes etapas de transformaciones y desarrollo en este tipo de Educación y su Pedagogía.

A partir de las ideas de los más destacados pedagogos de cada época se destaca la presencia de muchos de los presupuestos que sustentan hoy el desarrollo 


\section{Trabalhonecessário}

Issn: 1808 - 799X

ano 13, número 22 - 2015

de una Pedagogía de la ETP en Cuba; en especial su visión sobre la Educación General y Técnico-Profesional del trabajador, su cultura general mediante la unidad del estudio con el trabajo, de la educación con la actividad económica y las profundas concepciones de sus raíces humanistas y revolucionarias que tienen total vigencia la ETP cubana actual y en los postulados de su Pedagogía.

Los resultados de las investigaciones que hemos realizado en el ámbito de la Educación Técnica y Profesional han aportado referentes teóricos y metodológicos básicos que orientan las transformaciones de la práctica educativa. Sin embargo, aun cuando han permitido desde lo teórico-metodológico una acertada sistematización de los conocimientos y una adecuada atención a la práctica, se necesita fortalecer el sistema teórico científicamente fundamentado de la Pedagogía de la ETP, para mostrar su carácter científico y reconocer su lugar como rama de la Pedagogía.

Se puede evidenciar la existencia de problemas en la argumentación científica de los hechos y fenómenos inherentes a la Educación Técnica y Profesional, al desarrollo técnico-profesional integral de los obreros y al proceso de su formación técnico-profesional. Se pone de manifiesto una contradicción esencial entre el nivel actual de conocimientos pedagógicos y la necesidad de dirigir científicamente el proceso educativo en correspondencia con las tradiciones pedagógicas y con las tendencias actuales y perspectivas de la Educación Técnica y Profesional. Como una rama de la Pedagogía, a la Pedagogía de la ETP le corresponde aportar el sistema categorial y los métodos científico-investigativos que posibiliten el estudio y transformación del proceso educativo continuo de las trabajadoras y trabajadores cubanos.

El desarrollo de la economía socialista, unido a las transformaciones que ha sufrido el subsistema de la ETP para dar respuesta a las necesidades educativas y de la producción, demuestran la plena vigencia y lugar que tiene y tendrá la 


\section{Trabalhonecessário}

Issn: 1808 - 799X

ano 13, número 22 - 2015

educación técnica y profesional en el futuro, y por lo tanto, la necesidad del desarrollo de una Pedagogía de la ETP cubana que prepare a los profesores, directivos y personal de las entidades laborales para transformarla y elevarla a estos tiempos, para ver el futuro y adelantarse a él.

\section{Referencias}

ABREU REGUEIRO, R. Un Modelo de la Pedagogía de la Educación Técnica y Profesional en Cuba. Tesis en opción al Grado Científico de Doctor en Ciencias Pedagógicas, Universidad de Ciencias Pedagógicas "Enrique José Varona", La Habana, 2004.

BERMÚDEZ MORRIS, R. Y L.M. PÉREZ MARTíN. Modelo de los procesos formativos en el primer año del Curso Regular Diurno de las especialidades técnicas de la Licenciatura en Educación. Resultado del proyecto de investigación "Modelo para la dirección de los procesos formativos en el primer año del Curso Regular Diurno de la Licenciatura en Educación", asociado al Programa Ramal \# 8 del MINED. UCP "Héctor Alfredo Pineda Zaldívar". La Habana, 2010.

BERNAL ALEMANY, R. Particularidades del proceso educativo en los centros de la ETP. ICCP, La Habana. 1987 (Tesis doctoral)

CARTALLA COTA, P. José de la Luz y Caballero y la Pedagogía de su época, La Habana, 1989.

CASTRO RUZ, Fidel. Discurso pronunciado el 18 de diciembre de 1966 en la graduación de los primeros 425 técnicos del Consejo del Plan de la Enseñanza Tecnológica de Suelos, Fertilizantes y Ganadería. La Habana. http://www.cuba.cu/gobierno/discursos/1966/esp/f181266e.html

LEÓN GARCÍA, M. Modelo teórico de la integración Escuela Politécnica - Mundo Laboral en la formación de profesionales de nivel medio. ISPEJV. La Habana, 2003 (Tesis Doctoral).

PRIMER CONGRESO DEL PARTIDO COMUNISTA DE CUBA. Tesis y Resoluciones. Editorial de Ciencias Sociales, La Habana, 1975.

RAMIREZ DE LA CRUZ, 0. Discurso pronunciado el 30 de Julio en el Centro Tecnológico Frank País. La Habana, s/f.

RIVAS RIVAS, Hilda Y RIVAS RIVAS, Olga. La enseñanza y el aprendizaje de los oficios en Cuba durante los tres primeros siglos de colonización como una forma no escolarizada de educación. ISPETP, La Habana, 1992 (Informe de Investigación). 


\section{Trabalhonecessário}

Issn: 1808 - 799X

ano 13 , número $22-2015$

VARONA, Enrique José. Trabajos sobre educación y enseñanza. La Habana, Comisión Nacional Cubana de la UNESCO, 1961, p.17.

Recebido em 28 de novembro de 2015.

Aprovado em 08 de dezembro de 2015. 